\title{
Model-observation comparison study of multiple polar cap arcs
}

\author{
L. Zhu, C. E. Valladares, ${ }^{1}$ J. J. Sojka, R. W. Schunk, and D. J. Crain \\ Center for Atmospheric and Space Science, Utah State University, Logan
}

\begin{abstract}
A quantitative model-observation comparison of multiple polar cap arcs has been conducted by using a time-dependent theoretical model of polar cap arcs. In particular, the electrodynamical features of multiple polar cap arcs with various spacings are simulated and the results are compared with the images obtained from the All-Sky Intensified Photometer at Qaanaaq. The results show that the observed and simulated arcs are quite similar, both spatially and temporally. The results support the theory proposed by Zhu et al. [1993a, 1994b] that the structure of polar cap arcs is mainly determined by the magnetosphere-ionosphere (M-I) coupling processes and that the spacing of multiple polar cap arcs is closely related to the hardness of the primary magnetospheric precipitation. It is found that for the multiple polar cap arcs with both narrow and wide spacings, the associated field-aligned currents are mainly closed by Pedersen currents. It is also found that a hard precipitation can lead to a highly structured secondary arc because of the nonlinear M-I coupling processes.
\end{abstract}

\section{Introduction}

Polar cap arcs are the auroral arcs seen at very high geomagnetic latitudes $\left(>80^{\circ}\right)$ that extend from $100 \mathrm{~km}$ up to the size of the whole polar cap and that are aligned parallel to the Sun-Earth direction [Davis, 1960; Nielson et al., 1990]. The arcs are mainly observed during northward interplanetary magnetic field (IMF) and quiet magnetic conditions. Some of the arcs are very bright and luminous and cross the entire polar cap from the dayside to the nightside of the auroral oval to form a pattern that resembles the Greek letter "theta" [Frank et al., 1986; Nielsen et al., 1990]. Other arcs are confined to the polar cap and are relatively weak, and these tend to be either in the evening or morning sectors of the polar cap [Weber and Buchau, 1981; Carlson et al., 1984; Rich et al., 1990]. Some observations suggest that the Sun-aligned arcs at high latitudes are in regions of open field lines because polar rain electrons are sometime observed adjacent to and outside of the arcs [Hardy et al., 1982]. However, in other observations, the precipitating electrons have the characteristics of the plasma sheet or plasma sheet boundary layer, suggesting that the arcs are on closed field lines [Peterson and Shelley, 1984; Obara et al., 1988]. It has been confirmed by many observations that the arcs are associated with upward field-aligned currents carried by energetic precipitating electrons, with the energy range extending from a few hundred $\mathrm{eV}$ to a few $\mathrm{keV}$ [Hardy et al., 1982; Valladares and Carlson, 1991].

More recently, a series of campaigns conducted under the auspices of the CEDAR High Latitude Plasma Structure (HLPS) Working Group have accumulated a wealth of new information on polar cap arcs and have extended our observations from the

\footnotetext{
IInstitute for Space Research, Boston College, Newton, Massachusetts.

Copyright 1996 by the American Geophysical Union.
}

large-scale, steady state features to the more dynamical, mesoscale features of polar cap arcs. The new observations have provided information on, for example, the dawn-dusk motion and the multiple fine structure of polar cap arcs [Valladares et al., 1994], the sudden rotation of the adjacent convection prior to the breakup of polar cap arcs [Berg, 1992], and the field-aligned depletions of $F$ region plasma [Doe et al., 1992].

Compared to the observations, theoretical studies of polar cap arcs, especially quantitative studies, are still in an early stage. On the basis of S3-2 data, Burke et al. [1982] proposed a model for polar cap arcs that occur on open field lines. By introducing the concept of a multiple bifurcation of magnetic field lines, Kan and Burke [1985] constructed a model of polar cap arcs and proposed that theta aurora can occur on closed field lines and that the less intense polar cap arcs can occur either on closed or open field lines. More recently, Chiu [1989] formulated a model in which polar cap arcs are assumed to be on open field lines, and he showed that the formation of polar cap arcs is due to the current response to a mesoscale velocity shear structure in the ionosphere. Because these theoretical models are either qualitative, semiquantitative, or steady state models, they cannot be used to study the dynamical features associated with both the temporal evolution and the fine spatial structure of polar cap arcs. Also, these models cannot be used in a detailed quatitative comparison with observations, which is essential for furthering our understanding of the dynamics of polar cap arcs.

Recently, we have conducted a series of polar cap arc studies using a time-dependent magnetosphere-ionosphere (M-I) coupling model [Zhu et al., 1993a, b; Sojka et al., 1994; Zhu et al., 1994a, b]. These studies have provided satisfactory "theoretical" explanations for a number of important issues concerning polar cap arcs, which include the active role of the ionosphere for the formation of the arcs, the appearance of multiple arcs, and the physical parameters determining the spacing of multiple arcs.

In this paper, we present the results obtained in a detailed quantitative comparison of the M-I coupling model with 
measurements. In past years, several CEDAR-HLPS campaigns have led to a very extensive observational database of polar cap arcs, including data from all-sky cameras, sounding rockets, incoherent scatter radars, digisondes, etc., and many new features of polar cap arcs have been observed during these campaigns. To quantitatively explain the observed new features and study the associated electrodynamical processes, a series of model-observation comparison studies with the focus on these new features would be logical next steps. Since the M-I coupling model of polar cap arcs is time-dependent and electrodynamical, the model is particularly suited for comparisons with such a database. Here we are interested in comparing the model predictions for electrodynamical features of multiple polar cap arcs with various spacings to the images obtained from the All-Sky Intensified Photometer at Qaanaaq.

\section{Observations}

The images used in this model-observation study were obtained using the All-Sky Intensified Photometer (ASIP) located near the Greenlandic Village of Qaanaaq $\left(77.5^{\circ} \mathrm{N}\right.$, $69.2^{\circ} \mathrm{W}$ ). The Qaanaaq ASIP started operation in October 1983, and since then it has collected images of the aurora and the airglow during the winter months when the moon was below the horizon. A new lens was installed in February 1988 to increase the ASIP's field of view to $180^{\circ}$. The camera routinely alternates between $427.8-\mathrm{nm}$ and $630.0-\mathrm{nm}$ filters completing a two-filter cycle every $2 \mathrm{~min}$. The images, together with a visual display of the clock, are stored on film media. The initial image processing consists of a CCD-based digitization of the film and an identification of the boundaries of the camera field of view and an L-shaped mark that points to the north and east cardinal directions. When these patterns are successfully detected, appropriate transformations are performed for each pixel of the images, converting each image to a geographic or a corrected geomagnetic (CG) coordinate system representation.

Multiple polar cap arcs have been found in many all-sky camera images taken at Qaanaaq [e.g., Valladares et al., 1994] as well as in other observations. During 11 years of operations at the Qaanaaq station, about 0.5 million images

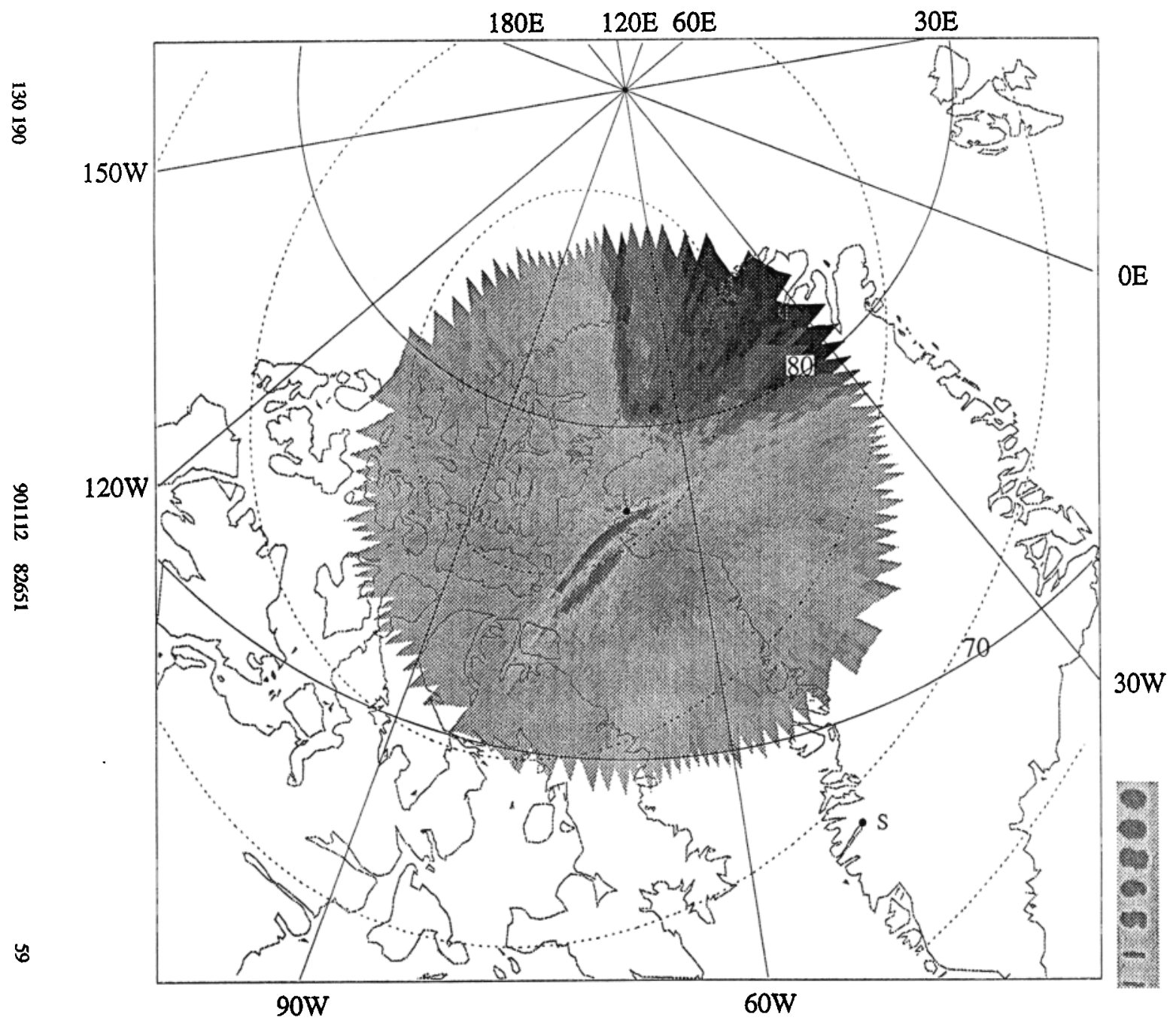

Figure 1. An image of multiple polar cap arcs taken by the All-Sky Intensified Photometer at Qaanaaq at 0826 UT, November 12, 1990. 
were taken and about $30 \%$ of them have polar cap arcs. Among the images having polar cap arcs, at least $80 \%$ of them show the multiple arc structures. The development of multiple polar cap arcs is quite dynamical. In about $85 \%$ cases of multiple polar cap arcs, the arcs with a multiple structure did not appear simultaneously. It is quite common that new arcs develop near the arc that appear in a early time to form multiple structures. The locations of individual arcs are not spatially stable, and they typically move in a dawn-dusk direction with a speed that ranges from tens of meters per second to several hundred meters per second. The typical spacing of the structured arcs can range from tens of kilometers to a few hundred kilometers. This paper is not to touch the details of the above statistical features of multiple polar cap arcs. Instead, we choose two typical cases of multiple arcs based on the above statistical results and perform model-observation comparison study to test the model predictions and reveal more dynamical features of the arcs.

Figure 1 shows an image taken by the all-sky camera at Qaanaaq at 0826 UT, November 12, 1990. Clearly, the polar cap arcs are structured and the edge-to-edge spacing (the width of the dark region between two arcs) is found to be about 40 $\mathrm{km}$. These polar cap arcs are not the transpolar arcs, instead, they are localized and confined to the morning sector of the polar cap.

\section{Theoretical Model}

The modeling of multiple polar cap arcs was conducted by using the time-dependent M-I coupling model developed by Zhu et al. [1993a]. The mathematical formulation of the model will not be discussed in this paper and the interested readers are referred to the Zhu et al. [1993a] paper. A key feature of this M-I coupling model of polar cap arcs is that the arcs are not treated as phenomena merely controlled by the magnetosphere, with the ionosphere only acting as a passive load. Instead, the polar cap arcs are treated as the processes that are coherently determined by the dynamics in both the ionosphere and magnetosphere.

Initially, a magnetospheric shear flow carried by Alfvén waves propagates towards the ionosphere. The significance of the assumed initial magnetospheric shear flow in the model has been discussed by Zhu et al. [1993a] and is not repeated here. The downward propagating Alfvén waves are partially reflected from the ionosphere and then bounce back and forth between the ionosphere and magnetosphere. The nature of the wave reflections depends on both the conditions in the ionosphere and magnetosphere. The propagating Alfven waves carry both upward and downward field-aligned currents. The precipitating electrons associated with upward fieldaligned currents enhance the conductivity in the ionosphere. The modified ionospheric conductivity launches secondary Alfvén waves towards the magnetosphere. The upward propagating Alfven waves, which consist of the reflected waves and the secondary Alfvern waves launched by the temporal change of the ionospheric conductivity, carry the ionospheric information back to the magnetosphere, thus reflecting the active ionospheric role in the dynamics of the M-I coupling process. The whole process is transient, during which all physical quantities in the ionosphere change selfconsistently in time, and subsequently, polar cap arcs develop. Because of the finite conductivity in the ionosphere, the temporal variation of the Alfvern waves in the coupled M-I system diminishes with time, and the M-I system, as well as the development of polar cap arcs, approach an asymptotic steady state after several bounce periods.

One important issue that need to be addressed here is the treatment of the relationship between the Alfven waves and the conductivity enhancement caused by precipitations. Due to the great variation of the Alfven speed along the magnetic field lines, the resonance between the precipitating particles and the Alfven waves may not be able to sustain all the time. To avoid the complicated wave-particle interaction component in the model, we use the following simplified approach to tackle the issue. On the basis of MHD theory, enhanced $E$ field associated with Alfvén waves leads to an enhanced field-aligned current. By assuming a linear relationship [Fridman and Lemair, 1980], we connect the upward field-aligned current and the field-aligned potential drop. The increasing potential drop along magnetic field lines causes the energization of the current carriers (we assume mainly electrons). By using continuity equation, we treat the conductivity enhancement caused by the energized precipitating electrons. In the reality, the picture for the connection between the Alfven waves and the resulted conductivity enhancement could be much more complicated.

The ionosphere is treated as a two-dimensional slab with an integrated conductivity. The $X$ axis measures the noonmidnight dimension and points to the dayside, and the $Y$ axis measures the dawn-dusk dimension and points to the duskside. The magnetosphere is a two-dimensional pseudoplane that is used as a boundary for the reflection of Alfvén waves. The magnetosphere and ionosphere are connected by magnetic field lines which are assumed to be perpendicular to the jonosphere. The communication between the magnetosphere and ionosphere is characterized by the bouncing of Alfven waves between the two regions, and both the upward and downward field-aligned currents are associated with the propagating Alfven waves. Initially, the large-scale ionospheric convection and conductivity are specified. These large-scale patterns are consistent with the overall solar, geomagnetic, and IMF conditions.

Interesting theoretical results concerning the electrodynamics of multiple polar cap arcs have recently been obtained with the M-I coupling model. With this model, Zhu et al. [1993a] showed that multiple polar cap arcs might not be due to multiple structures in the magnetospheric source region. Instead, they can be internally generated in the M-I system as a result of the self-consistent coupling of the magnetosphere and ionosphere. Sojka et al. [1994] found that the appearance of multiple polar cap arcs has a strong dependence on the magnitude of the large-scale ionospheric background convection $(E)$. With the same initial magnetospheric driver, they found that the number of the arcs increased with an increase in the strength of the background convection, while the spacing between the arcs remained constant. This dependence is "not" a linear effect in which the polar cap arcs simply scale with the magnitude of the background convection. The degree of striation, or multiple character, of the arcs increases rapidly from a single arc at $E<$ $20 \mathrm{mV} / \mathrm{m}$ to 5 arcs at $30 \mathrm{mV} / \mathrm{m}$. These results further indicate that the ionosphere plays an active role in the formation of multiple polar cap arcs and that the ionosphere dynamically responds to the magnetospheric driver. Expanding on the Sojka et al. [1994] results, Zhu et al. [1994b] reached a quatitative theoretical conclusion about the appearance and spacing of multiple polar cap arcs. They predicted that 


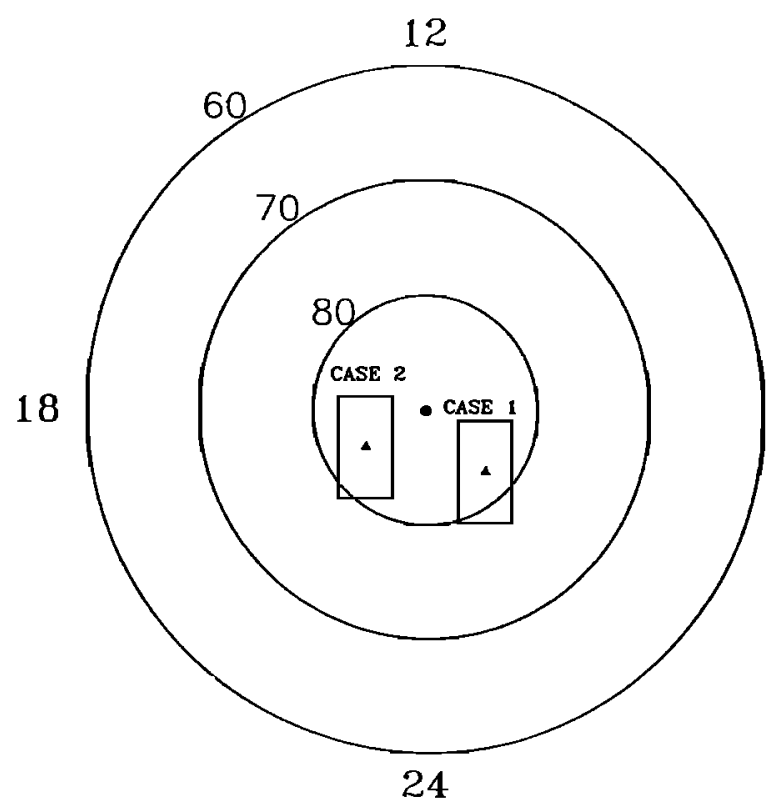

06

Figure 2. A schematic diagram showing the location of the ionospheric simulation domain in the polar cap. A magnetic latitude-magnetic local time (MLT) coordinate is used.

multiple polar cap arcs are more likely to occur when the large-scale ionospheric convection field is larger than 20 $\mathrm{mV} / \mathrm{m}$ and the background ionospheric Hall conductance is in the range of from 0.5 to 2 mho. When the large-scale convection is weak and the ionospheric conductance is high, a single arc is more likely to be seen in the polar cap. With a variable conductance ratio, it was found by Zhu et al. [1994b] that the spacing of multiple polar cap arcs is mainly determined by the hardness of the primary magnetospheric precipitation. A harder precipitation causes a wider spacing between individual arcs and vice versa. The edge-to-edge spacing between arcs varies from about $20 \mathrm{~km}$ to about $60 \mathrm{~km}$ when the ratio of the enhanced Hall and Pedersen conductances changes from 1 to 2 .

The above quantitative theoretical predictions concerning multiple polar cap arcs can be tested with the Qaanaaq data. By using model inputs that are consistent with the conditions when the observed multiple polar cap arcs occurred, we can compare the time-dependent electrodynamical features of both the simulated and observed arcs. If the corresponding spatial and temporal scales are largely consistent, that would confirm the theoretical predictions made by the M-I coupling model to zero-order. Also, the modeling results would provide a much more detailed picture of the electrodynamics of multiple polar cap arcs than the observations. The model yields information about the conductivity enhancement, the Joule heating, the connection between field-aligned currents and horizontal currents, the cross-flow associated with multiple polar cap arcs, etc. Such information would improve our understadings of the underlying physics of multiple polar cap arcs.

\section{Model-Observation Studies}

\section{Case 1: November 12, 1990}

The polar cap arcs observed on November 12, 1990 , occurred in the morning sector of the dark polar cap. The time period we are interested in is from 0822 to $0828 \mathrm{UT}$, during which the arcs went through the stages from breaking up to a quasi-steady state. During this time period, the IMF $B_{z}$ was positive $(\sim 1.5 \mathrm{nT})$ and $B_{y}$ was negative $(\sim-1.5 \mathrm{nT})$. Before this period, the IMF $B_{z}$ component had been positive for almost 24 hours, and after the period under study the IMF $B_{z}$ remained positive for another 24 hours. The $K p$ index was 1for almost 9 hours. This is a typical condition for the occurrence of polar cap arcs.

The location of the ionospheric simulation domain in the polar cap for case 1 is shown in Figure 2. The simulation domain is $1500 \mathrm{~km}$ long in the $X$ (midnight-noon) direction and $1000 \mathrm{~km}$ wide in the $Y$ (dawn-dusk) direction. The grid size is $30 \mathrm{~km}$ in the $X$ direction, and $10 \mathrm{~km}$ in the $Y$ direction. It should be noted that Figure 2 is just a schematic diagram and is not precisely scaled. With the M-I coupling model, we need to specify some initial conditions, which include the largescale background convection and background conductance patterns before the breakup of the polar cap arcs and the smallscale shear flow associated with the arcs. Unfortunately, for both observational periods in this study, there were either no DMSP data or the pass of DMSP was far away from the field of view of the all-sky camera. Therefore the DMSP satellite was not able to provide information on either the large-scale convection pattern or the small-scale shear flow associated with the arcs for the modelings. Hence we had to use

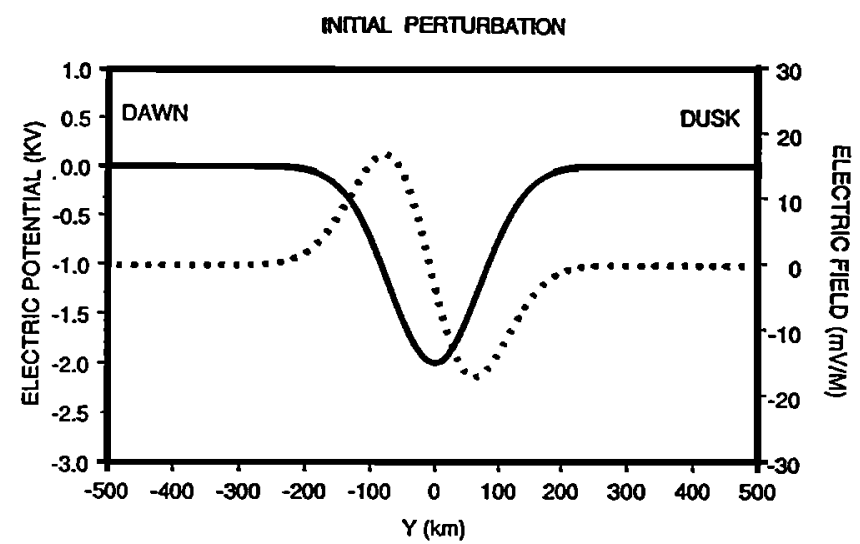

Figure 3. Potential distribution in the dawn-dusk cross section associated with the initial magnetospheric shear flow (solid) and the corresponding electric field distribution (dashed).

Figure 4. (left) A sequence of the images showing polar cap arcs starting at 0822 UT, November 12, 1990, with a 2-min interval and (right) the snapshots of the evolution of the field-aligned current distribution from the modeling. The dashed lines indicate upward field-aligısed currents. The ionospheric dimension of the images in the left column is about $1430 \mathrm{~km}$ in noon-midnight $(X)$ direction and about $1165 \mathrm{~km}$ in dawn-dusk (Y) direction. 

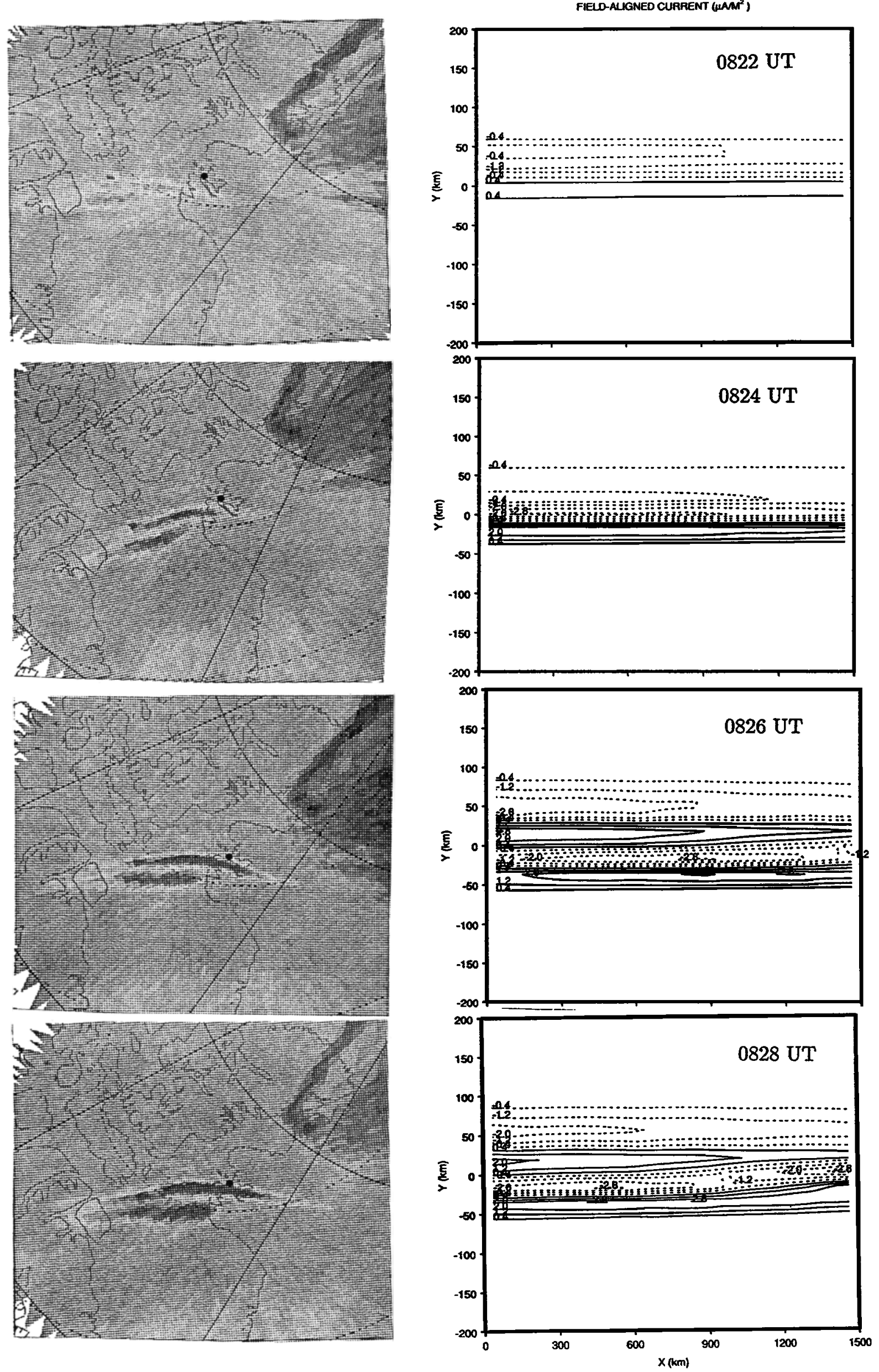
HALL CONDUCTANCE (MHO)

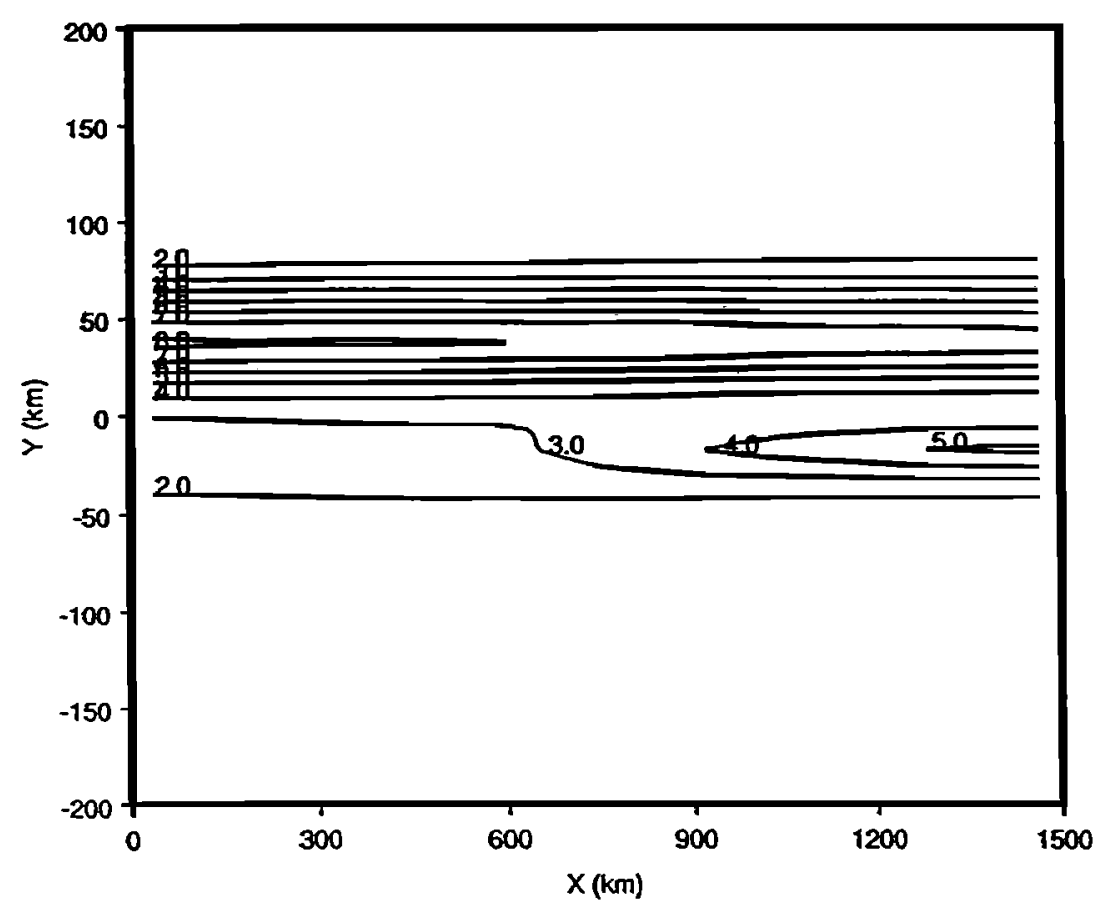

JOULE HEATING (ERG/CM² SEC)

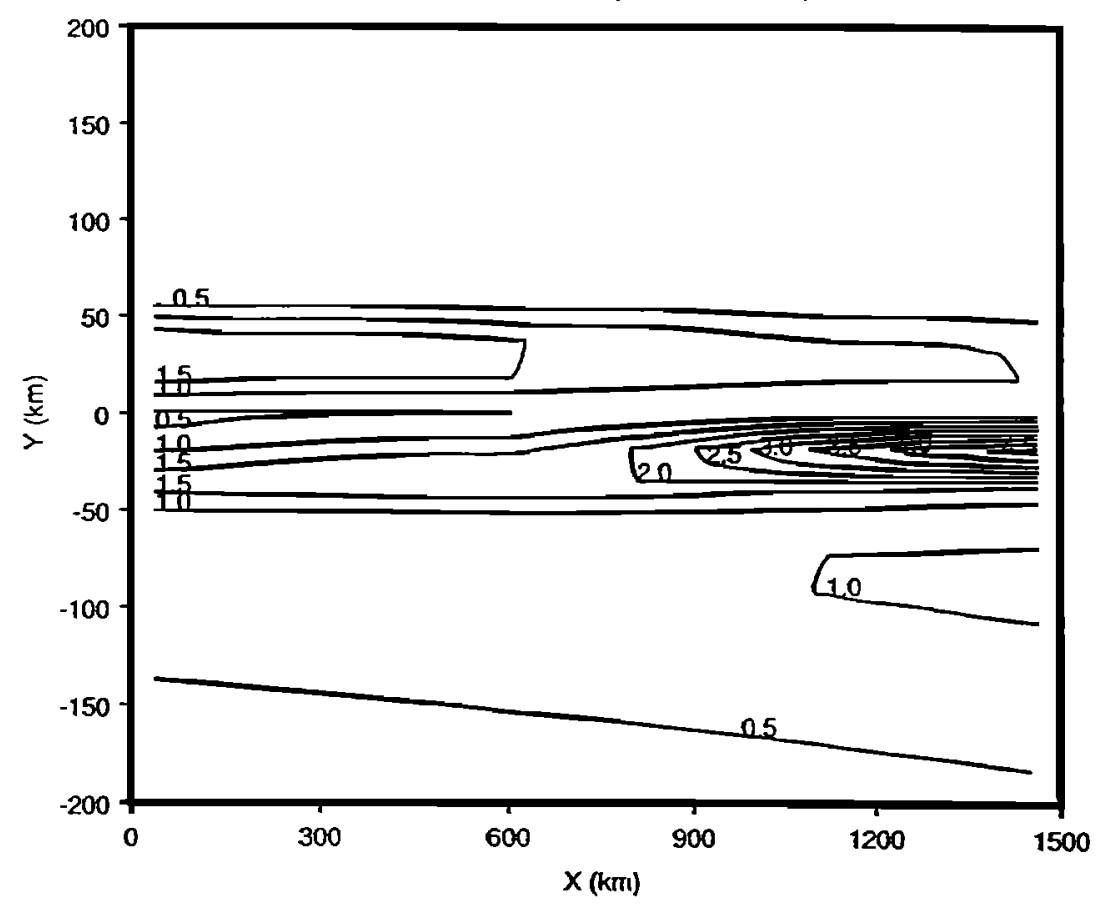

Figure 5. The distributions of the (top) enhanced Hall conductance and (bottom) Joule heating rate near the asymptotic steady state (0826 UT). No neutral wind effect is included in the calculation of the Joule heating rate.

statistical large-scale convection patterns for the IMF and geomagnetic conditions similar to those when the polar cap arcs occurred. On the basis of the Heppner and Maynard [1987] convection model, the large-scale background convection in the region where the arcs occurred for case 1 is basically uniform and antisunward. The background ionospheric conductance for the modelings was obtained from the conductivity model developed by Rasmussen et al. [1988] using the appropriate geomagnetic, solar, and seasonal conditions.

The M-I coupling model also needs the initial small-scale shear flow originated in the magnetosphere. Since $Z h u$ et al. [1994b] found that the appearance and spacing of "multiple" polar cap arcs are not determined by the spatial scale of the initial magnetospheric shear flow and they are determined by the M-I coupling processes, we used the same shear flow 
profiles for both of the cases we studied. The initial smallscale shear flow was assumed to be carried by a downward propagating Alfvén wave. It extended uniformly along the Sun-aligned direction ( $X$ direction) and had a "single" precipitation channel. The cross-section profiles of the electric field associated with the assumed initial small-scale shear flow (dashed line) and the corresponding electric potential (solid line) are shown in Figure 3.

It should be noted again that a guideline for our simulations is the theoretical results of Zhu et al. [1994b]. They found that the observed multiple polar cap arcs may not be due to multiple structures in the magnetosphere, but instead may primarily be determined by the coupled magnetosphereionosphere system in which the ionosphere plays an active role. The spacing of multiple polar cap arcs is mainly determined by the hardness of the primary magnetospheric precipitation. A harder precipitation causes a wider spacing between individual arcs and vice versa. Accordingly, for case 1 , in which the arcs have a narrow spacing, we assumed that the ratio between Hall and Pedersen conductances is 1.4, which reflects a relatively soft precipitation.

The simulation for case 1 started at $0818 \mathrm{UT}$, which is the time when the assumed downward propagating Alfvén wave hit the ionosphere. Figure 4 shows snapshots of the evolution of the field-aligned current distribution from the modeling (right column) and the corresponding time sequence of polar cap arc images (left column). From the images, starting at 0822 UT with a 2-min interval, we can see that the polar cap arcs started to appear at $0822 \mathrm{UT}$ and took about $6 \mathrm{~min}$ to reach a steady state. The arcs were structured with an edge-to-edge spacing
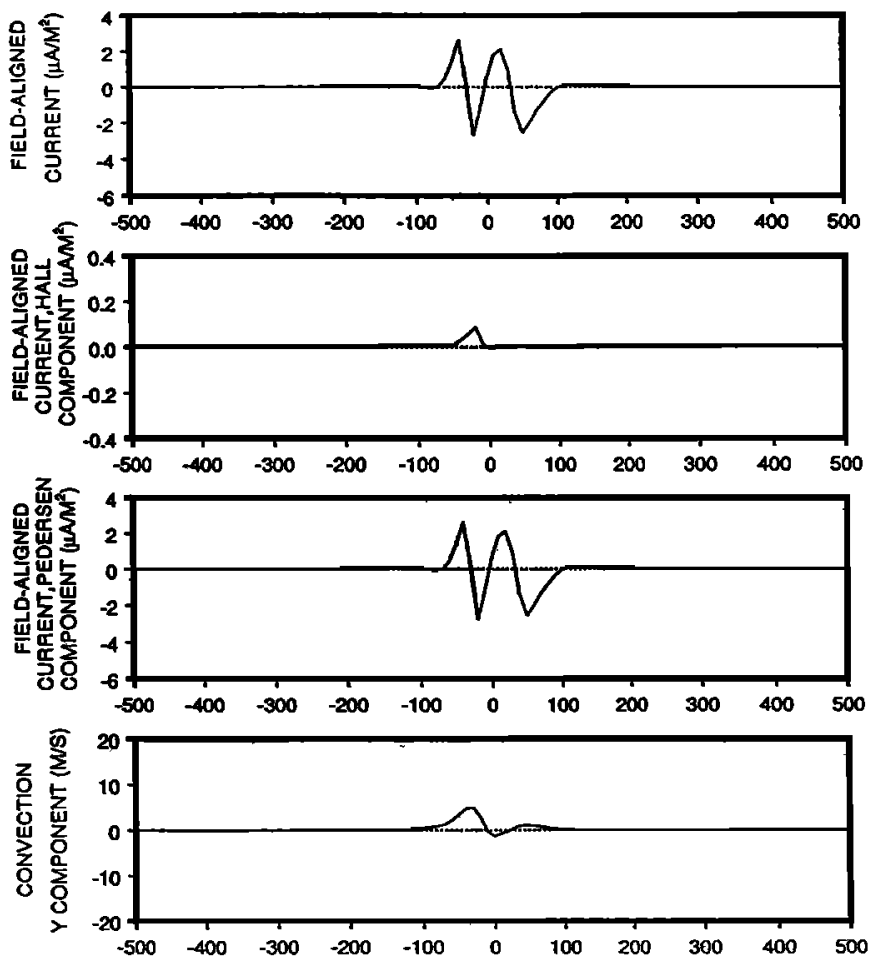

Figure 6. The cross-section profiles of the total fieldaligned current, the Hall and Pedersen components of the fieldaligned currents, and the cross flow associated with the polar cap arcs at 0826 UT from the modelings. The negative values of field-aligned current mean the upward field-aligned currents. (dark region between two arcs) of about $40 \mathrm{~km}$. The arcs were aligned mainly in the noon-midnight direction. The first plot of the modeled field-aligned currents is for $0822 \mathrm{UT}$, which is $4 \mathrm{~min}$ after the initial magnetospheric shear flow reaches the ionosphere. The successive plots are also at 2-min intervals. The dashed lines represent the upward field-aligned currents. Comparing the left and right columns, it is apparent that both the spatial and temporal features of the model predictions and measurements are quite similar except the intensity of the poleward arc at 0824 UT. These similarities indicate that our adopted initial conditions for the modeling are largely reasonable and that the M-I coupling model describes the appropriate physics associated with multiple polar cap arcs.

Figure 5 shows the distributions of the enhanced Hall conductance (top panel) and the Joule heating rate (bottom panel) at the asymptotic steady state (0826 UT). It can be seen that the Hall conductance is enhanced from about 0.5 mho (background conductance) to a peak conductance of 8 mho and that the enhanced conductance is structured. The Joule heating rate is also substantially increased and structured. It should be noted that there is no neutral wind effect in the calculation of Joule heating rate.

Figure 6 shows the cross-section profiles of the modeled total field-aligned current, the Hall and Pedersen components of the field-aligned currents, and the cross-flow associated with the polar cap arcs at 0826 UT, as obtained from the modelings. A negative value in the field-aligned current plots means upward current. These cross-section profiles clearly show the multiple structure of the polar cap arcs. Comparing the first three plots in Figure 5, we can see that the fieldaligned currents associated with multiple polar cap arcs are mainly closed by the Pedersen currents, and only a small part of the field-aligned currents is closed by the Hall currents. The Hall current contribution to the field-aligned currents is due to the small-scale conductivity gradient caused by the arc precipitation. It should be noted that if the ionosphere is in a steady state and the conductivity is uniform, there is no Hall current component of field-aligned currents. However, if the ionospheric system is temporally varying or the conductivity is nonuniform, the Hall current can make contribution to the field-aligned current. In principle, the closure of the ionosphere-originated field-aligned currents, which could include both Pedersen and Hall components, at the magnetosphere is achieved through the adjustment of magnetic field configuration in the magnetosphere. However, the detailed picture is still not clear and the mechanism for the current closure at the magnetosphere has not been selfconsistently treated in our model. The bottom panel shows that a cross flow exists in association with the multiple polar cap arcs, but the magnitude of the cross flow is small. It should be noted that this cross-flow results from the M-I coupling processes associated with the multiple arcs, since the initial magnetospheric shear flow is symmetric and does not contain a cross flow.

\section{Case 2: December 11, 1990}

The polar cap arcs observed on December 11, 1990, occurred in the evening sector of the dark polar cap. The time period we are interested in is from 2112 to 2118 UT. The IMF turned northward at about 1800 UT and remained northward until 0030 UT of the next day. During the period we are interested in, the $B_{z}$ was $\sim 5 \mathrm{nT}$ and $B_{v}$ was $\sim-1.5 \mathrm{nT}$, and $K p$ 

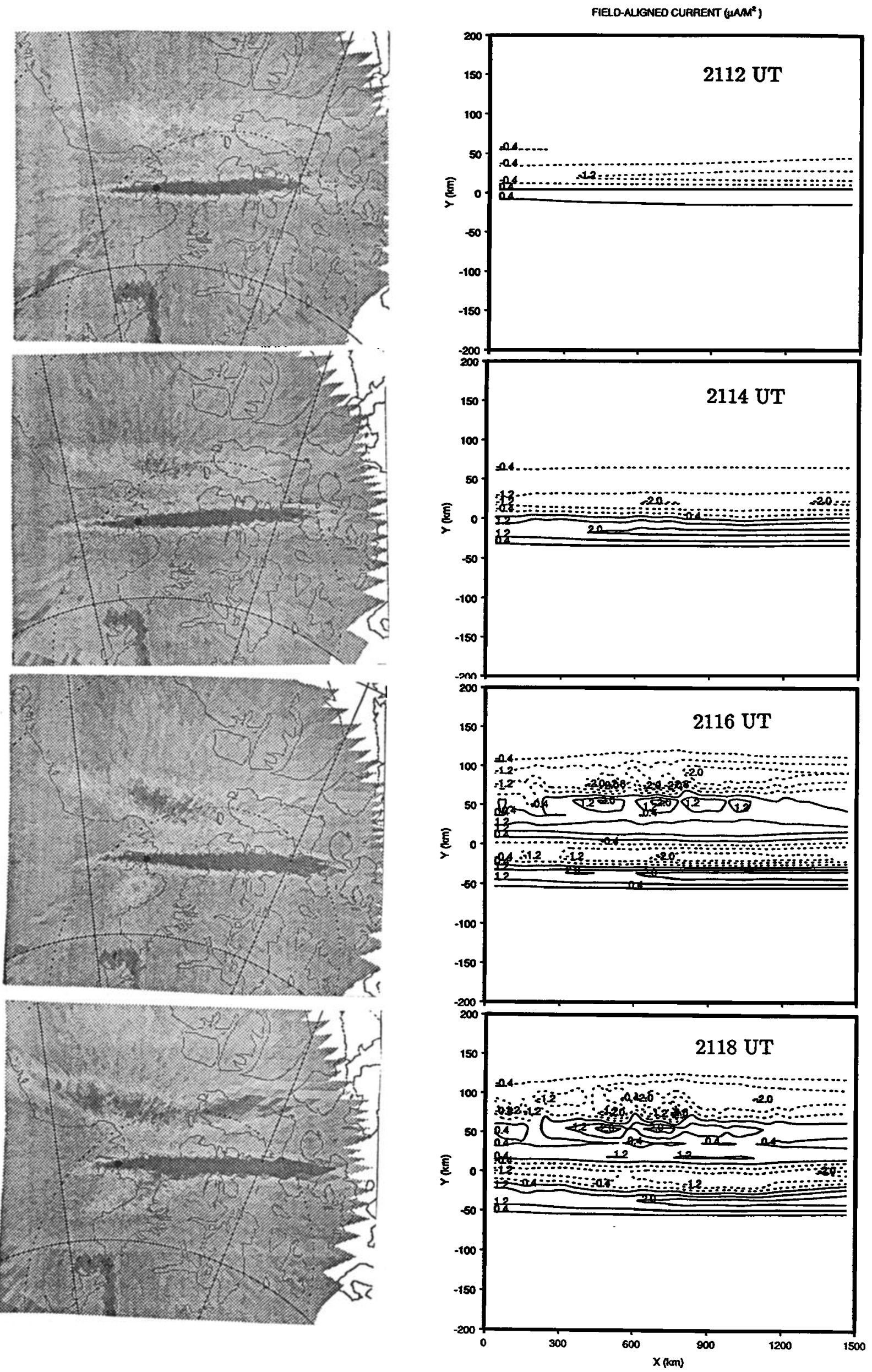
HAU CONDUCTANCE (MHO)

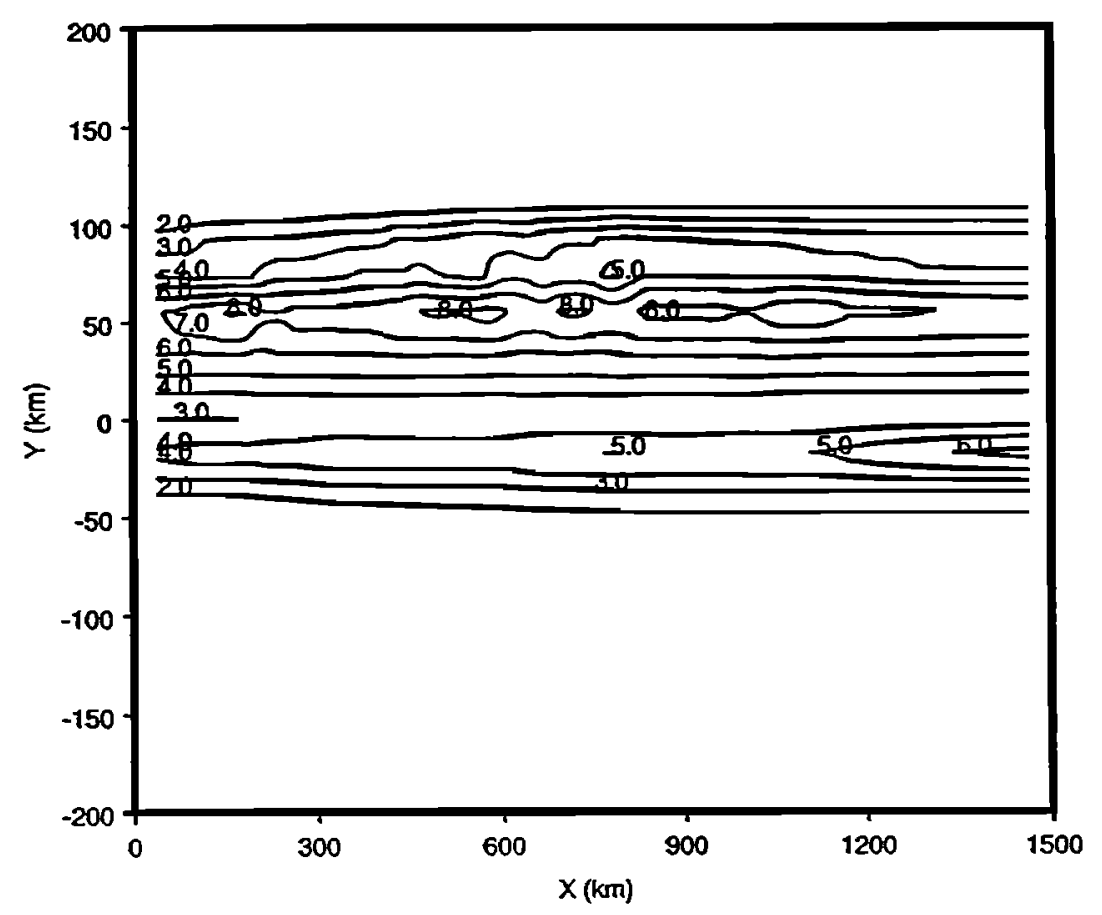

JOULE HEATING ( ERG/CM² SEC)

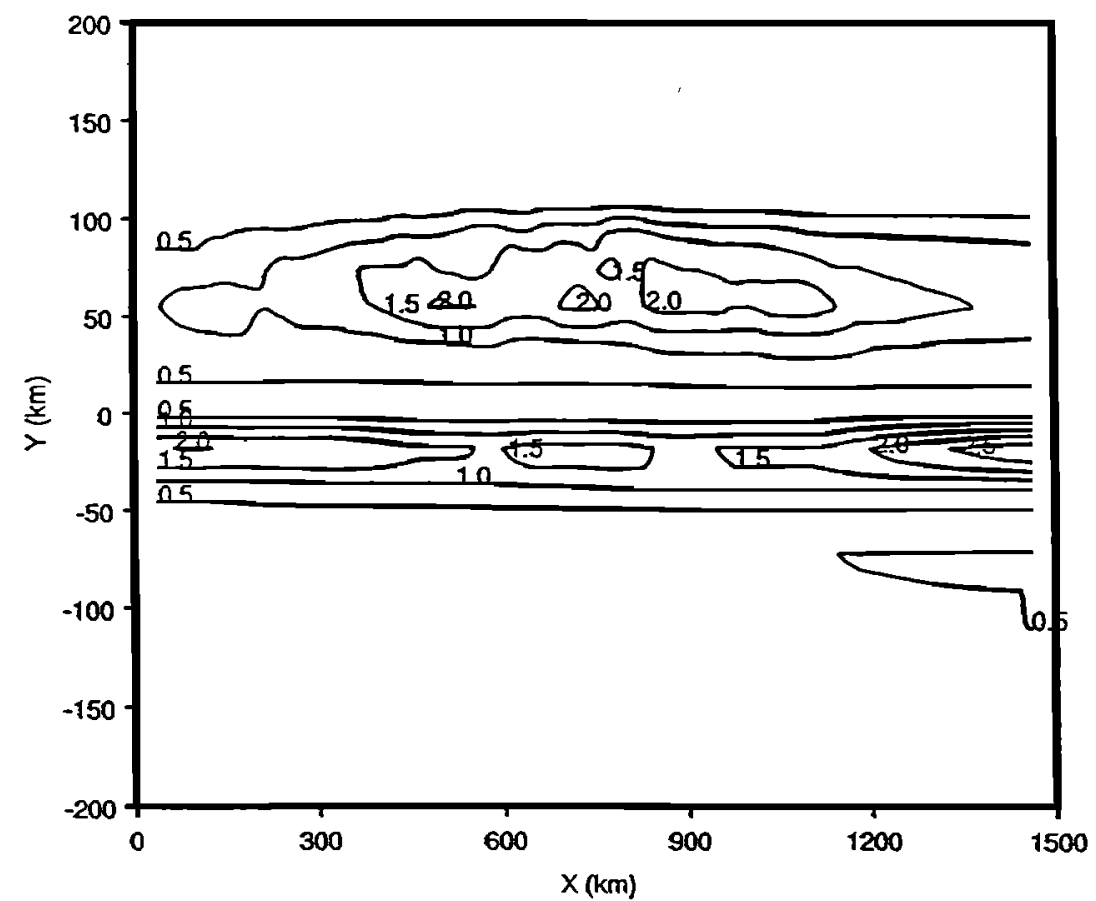

Figure 8. The distributions of the (top) enhanced Hall conductance and (bottom) Joule heating rate near the asymptotic steady state (2118 UT). No neutral wind effect is included in the calculation of the Joule heating rate.

Figure 7. (left) A sequence of images showing polar cap arcs starting at 2112 UT, December 11, 1990, with a 2 -min interval and (right) the snapshots of the evolution of the field-aligned current distribution from the modeling. 
was 1. This is also a typical condition for the occurrence of polar cap arcs. The location of the ionospheric simulation domain in the polar cap for case 2 is schematically shown in Figure 1.

For this case, the DMSP-F8 pass was about $1000 \mathrm{~km}$ away from the edge of the field of view of the all-sky camera. Therefore we again had to depend on statistical convection models for information about the large-scale background convection pattern. The Heppner and Maynard [1987] convection model shows that under the above specific IMF and geomagnetic condictions, the convection in the region where the multiple arcs in case 2 occurred is also basically uniform and antisunward. Then, by using the same magnetospheric shear flow and background ionospheric conductance as for case 1 , we conducted the modeling for case 2. The ratio of the Hall and Pedersen conductances for case 2 is 1.9 , which is different from that in case 1 and reflects a relatively harder precipitation.

The simulation for case 2 started at $2108 \mathrm{UT}$, which is the time when the assumed downward propagating Alfvén wave hit the ionosphere. Figure 7 shows snapshots of the evolution of the field-aligned current distribution from the modeling (right column) and the sequence of polar cap arc images (left column). The images starts at 2112 UT with a 2-min interval. The arc pattern consists of two arcs with an edge-to-edge spacing of about $90 \mathrm{~km}$, which is much wider than that in case 1. The arcs are again aligned mainly in the noon-midnight direction. It may be noticed that there is a significant bending associated with the equatorward arc at 2118 UT. This bending is due to the instrument effect which occurs when the altitude of the arc varies significantly along the arc and the arc is far away from the center of the field of view. The first plot of the modeled field-aligned currents is for $2112 \mathrm{UT}$, which is $4 \mathrm{~min}$ after the initial magnetospheric shear flow reaches the ionosphere. The successive plots are also at 2-min intervals. Again, a comparison of the left and right columns indicates that the two are quite similar, both spatially and temporally.

Special attention needs to be paid to the second arc. Zhu et al. [1993a] suggested that the nature of the primary and secondary arcs could be quite different. The primary arc is directly related to the magnetospheric driver. The appearance of the secondary arc and the spacing between the primary and secondary arcs are largely determined by the M-I coupling processes. Here, as a nonlinear response to a harder magnetospheric precipitation from the coupled M-I system, there is a wider spacing between the primary and secondary arcs. Also, the secondary arc shows fine structure feature. The feature of fine structure in the secondary arc from the simulation for the situation of hard precipitation is quite similar to that of the secondary arc in the images. This correspondence is a surprising finding. Further theoretical and modeling are needed to explore the underlying physics of this new feature.

Figure 8 shows the distributions of the enhanced Hall conductance (top panel) and the Joule heating rate (bottom panel) rear the asymptotic steady state (2118 UT). It can be seen that the Joule heating associated with the secondary arc is distributed. Figure 9 shows the cross-section profiles of the total field-aligned current, the Hall and Pedersen components of the field-aligned currents, and the cross-flow of the polar cap arcs at $2118 \mathrm{UT}$, as predicted by the modelings. From Figure 6, we can see that in the case of the arcs with a wide spacing, the field-aligned currents associated are also mainly
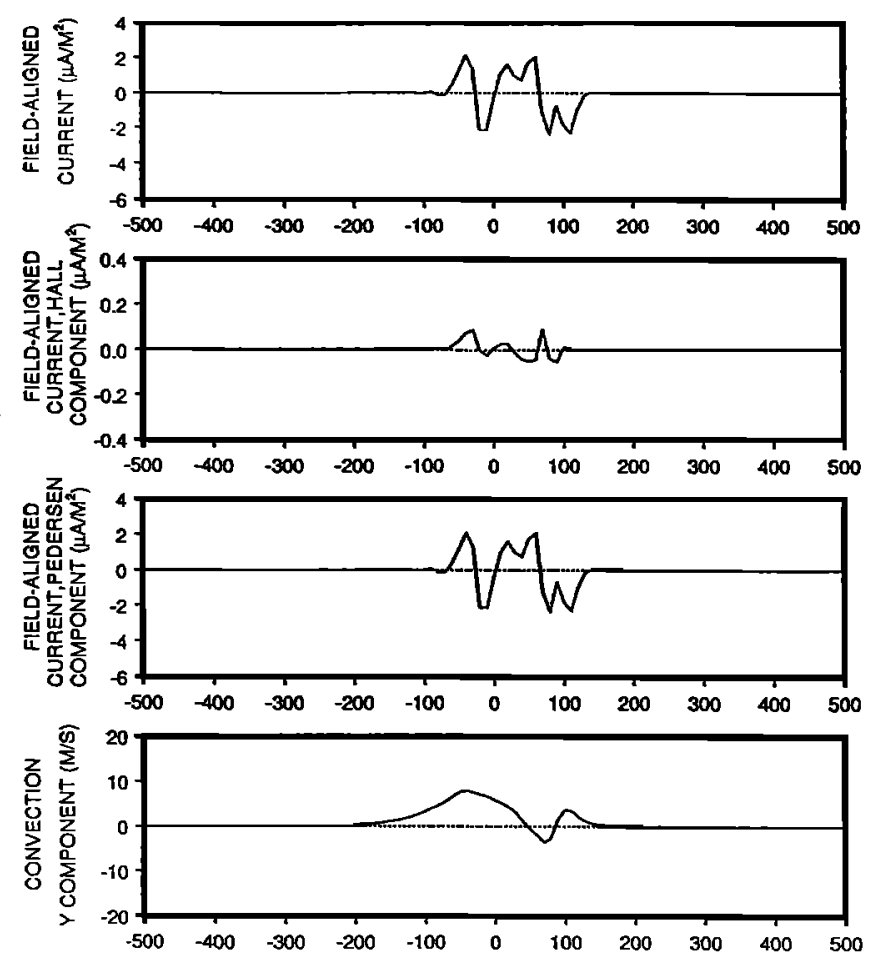

Figure 9. The cross-section profiles of the total fieldaligned current, the Hall and Pedersen components of the fieldaligned currents, and the cross flow associated with the polar cap arcs at 2118 UT from the modelings. The negative values of field-aligned current mean the upward field-aligned currents.

closed by Pedersen currents. In addition to the two distinct arcs, fine structure is clearly evident in the secondary arc. The bottom panel shows that there also exists a cross flow for the multiple polar cap arcs with a wide spacing, but the cross flow is considerably more distributed than that for the arcs with a narrow spacing, case 1 .

\section{Discussion and Conclusion}

For a first time, a quantitative model-observation comparison of multiple polar cap arcs has been conducted by using a time-dependent theoretical model of polar cap arcs and the initial results are encouraging. The arcs observed with the all-sky camera at Qaanaaq and the simulated ones are quite similar, both spatially and temporally. This direct comparison study of both temporal and spatial features of polar cap arcs is beyond the capability of previous theoretical models. The key difference between the two cases we considered was the arc spacing. Zhu et al. [1994b] predicted that the spacing of multiple polar cap arcs is mainly determined by the hardness of the primary magnetospheric precipitation. A harder precipitation causes a wider spacing between individual arcs and vice versa. Our modelobservation study confirms this prediction. However, the prediction can be further tested in a study by combining precipitation data from DMSP satellites with ground-based image data. The precipitation observation would define the spectral hardness that has been used as an assumed input parameter in this study.

The initial magnetospheric shear flow for both cases under 
study was a simple symmetric convection shear with a single precipitation channel, but we successfully produced arcs that had multiple structures which were very similar to the observed ones. This further indicates that the observed multiple polar cap arcs may not be due to multiple structures in the magnetosphere but instead may primarily be determined by the coupled magnetosphere-ionosphere system in which the ionosphere plays an active role.

Our results show that for the multiple polar cap arcs with both narrow and wide spacings, the associated field-aligned current are mainly closed by Pedersen currents, and the Hall currents only make a small contribution. A cross flow occurs for both type of multiple arcs, but the cross flow for the arcs with a wide spacing is more spread.

In the observed arcs of case 2, the secondary arc is highly structured. To our surprise, the model developed a secondary arc with a similar fine structure. The fine structure in secondary arc for the multiple arcs with wide spacing (or hard precipitation) is a new finding. We believe this is due to the nonlinear M-I coupling process, but more theoretical analyses and modelings are needed to understand the detailed underlying physics,

This work is just one of the coordinated endeavors of the HLPS campaigns to understand the plasma structures observed in the high-latitude regions. In past years, CEDAR-HLPS campaigns have led to a very extensive observational database of polar cap arcs and many new features have been discovered. This makes quatitative model-observation studies very feasible, and they are essential for improving our understanding of polar cap arcs.

Acknowledgment. The work at Utah State University was supported by NASA grant NAG5-1484 and by NSF grants ATM-9308163 and ATM-93-02165. The work at Boston College was supported by Phillips Laboratory contract F19628-90-K-007 and by NSF grant ATM-9404088. We thank the Danish Commission for Scientific Research in Greenland for permission to conduct ground experiments at Qaanaaq under continuing project A16-91. We thank Katsura Fukui and Hugh Gallagher for helping to digitize the images presented here. We thank two referees for their very constructive comments and suggestions.

The Editor thanks F. J. Rich and another referee for their assistance in evaluating this paper.

\section{References}

Berg, G. A., In-situ and ground-based observations of Sun-aligned arcs, paper presented at the Joint Workshop for CEDAR HLPS/STEP GAPS, NSF, Peaceful Valley, Colo., June 1992.

Burke, W. J., M. S. Gussenhoven, M. C. Kelley, D. A. Hardy, and F. J. Rich, Electric and magnetic characteristics of discrete arcs in the polar cap, J. Geophys. Res., 87, 2431, 1982.

Carlson, H. C., Jr., V. B. Wickwar, E. J. Weber, J. Buchau, J. G. Moore, and $\mathrm{W}$. Whiting, Plasma characteristics of polar cap $F$-layer arcs, Geophys. Res. Lett., 1, 895, 1984.

Chiu, Y. T., Formation of polar cap arcs, Geophys. Res. Lett., 16, 743, 1989.

Davis, T. N., The morphology of the polar aurora, J. Geophys. Res., 65, 3497, 1960.
Doe, R., M. Mendillo, J. Vickrey, M. Ruohoniemi, and R. Greenwald, Coordinated convection measurements in the vicinity of auroral cavities and arcs, paper presented at the Joint Workshop for CEDAR HLPS/STEP GAPS, NSF, Peaceful Valley, Colo., June 1992.

Frank, L. A., et al., The theta aurora, J. Geophys. Res., 91, 3177, 1986.

Fridman, M., and J. Lemaire, Relationship between auroral electron fluxes and field-aligned electric potential difference, $J$. Geophys. Res., 85, 664, 1980.

Hardy, D. A., W. J. Burke, and M. S. Gussenhoven, DMSP optical and electron measurements in the vicinity of polar cap arcs, J. Geophys. Res., 87, 2413, 1982.

Heppner, J. P., and N. C. Maynard, Empirical high-latitude electric field models, J. Geophys. Res., 92, 4467, 1987.

Kan, J. R., and W. J. Burke, A theoretical model of polar cap auroral arcs, J. Geophys. Res., 90, 4171, 1985.

Nielsen, E., J. D. Craven, L. A. Frank, and R. A. Heelis, Ionospheric flows associated with a transpolar arc, J. Geophys. Res., 95, 21169. 1990.

Obara, T., M. Kitayama, T. Mukai, N. Kaya, J. S. Murphree, and L. L. Cogger, Simultaneous observations of Sun-aligned polar cap arcs in both hemispheres by EXOS-C and Viking, Geophys. Res. Lett., 15, $713,1988$.

Peterson, W. K., and E. G. Shelley, Origin of the plasma in a crosspolar cap auroral feature (theta aurora), J. Geophys. Res.. 89, 6729, 1984.

Rasmussen, C. E., R. W. Schunk, and V. B. Wickwar, A photochemical equilibrium model for ionospheric conductivity, J. Geophys. Res., 93, 9831, 1988.

Rich, F. J., D. A. Hardy, R. H. Redus, and M. S. Gussenhoven, Northward IMF and patterns of high-latitude precipitation and fieldaligned currents: The February 1986 storm, J. Geophys. Res., 95, $7893,1990$.

Sojka, J. J., L. Zhu, D. J. Crain, and R. W. Schunk, Effect of high latitude ionospheric convection on Sun-aligned polar cap arcs, $J$. Geophys. Res., 99, 8851, 1994.

Valladares, C. E., and H. C. Carlson, The electrodynamic, thermal, and energetic character of intense sun-aligned arcs in the polar cap, $J$. Geophys. Res., 96, 1379, 1991.

Valladares, C. E., H. C. Carlson, and K. Fukui, Interplanetary magnetic field dependency of stable Sun-aligned polar cap arcs, J. Geophys. Res., 99, 6247, 1994.

Weber, E. J., and J. Buchau, Polar cap F-layer auroras, Geophys. Res. Lett., 8, 125, 1981.

Zhu, L., J. J. Sojka, R. W. Schunk, and D. J. Crain, A time-dependent model of polar cap arcs, J. Geophys. Res., 98, 6139, 1993a.

Zhu, L., J. J. Sojka, R. W. Schunk, and D. J. Crain, Influence of horizontal inhomogeneity in the ionosphere on the reflection of Alfvén waves, Geophys. Res. Lett., 20, 313, 1993b.

Zhu, L., J. J. Sojka, R. W. Schunk, and D. J. Crain, Theoretical study of polar cap arcs: Time-dependent model and its applications, Radio Sci., 29, 283, 1994a.

Zhu, L., J. J. Sojka, R. W. Schunk, and D. J. Crain, Model study of multiple polar cap arcs: Occurrence and Spacing, Geophys. Res. Lett.. 21, 649, 1994b.

D. J. Crain, R. W. Schunk, J. J. Sojka, and L. Zhu, Center for Atmospheric and Space Sciences, Utah State University, Logan, UT 84322-4405. (zhu@theory.cass.usu.edu)

C. E. Valladares, Institute for Space Research, Boston College, Newton, MA 02159.

(Received September 28, 1994; revised September 1, 1995; accepted September 1, 1995.) 